\title{
EXTREMELY LOW THERMAL CONDUCTIVITY OF AMORPHOUS ICE: RELEVANCE TO COMET EVOLUTION
}

\author{
A. KouChI AND J. M. GREENBERG \\ Laboratory Astrophysics, Huygens Laboratory, University of Leiden, Niels Bohrweg 2, 2333 CA Leiden, The Netherlands \\ T. YамAMOTO \\ Institute of Space and Astronautical Science, Yoshinodai, Sagamihara, Kanagawa 229, Japan \\ AND \\ T. MuKaI \\ Department of Earth Sciences, Kobe University, Nada, Kobe 657, Japan \\ Received 1991 September 26; accepted 1992 January 15
}

\begin{abstract}
The thermal conductivity of very slowly deposited amorphous ice derived from experimental results is shown to be a factor of $10^{-4}$ to $10^{-5}$ less than hitherto estimated. Using the exceedingly low value of the thermal conductivity of comets deduced from the amorphous ice properties leads to the expectation that internal heating of comets is negligible below the outer several tens of centimeters.
\end{abstract}

Subject headings: comets: general

\section{INTRODUCTION}

The importance of knowing the thermal conductivity, $\kappa$, of vapor-deposited amorphous solid water $\left(\mathrm{a}-\mathrm{H}_{2} \mathrm{O}\right)$ for predicting the thermal evolution of comets has been widely acknowledged (e.g., Klinger 1980; Smoluchowski 1985; Espinasse et al. 1991). However, there has been no previous direct measurement available, so that most of the discussions on comet evolution have been based on a theoretical estimation of $\kappa$ by Klinger (1980). Slow vapor deposition of $\mathrm{a}-\mathrm{H}_{2} \mathrm{O}$ is relevant to the formation of interstellar dust ice mantles (d'Hendecourt, Allamandola, \& Greenberg 1985) and therefore to comets as aggregated interstellar dust (Greenberg 1982). On the basis of our new experimental results and a reanalysis of data found in the literature, we have arrived at a new estimate of the $\kappa$ for $\mathrm{a}-\mathrm{H}_{2} \mathrm{O}$. This will make it possible to apply the results to thermal evolution of comets (Greenberg, Yamamoto, \& Xing 1992).

\section{EXPERIMENTAL}

A thin film of $\mathrm{H}_{2} \mathrm{O}$ ice was deposited very slowly onto a metal substrate at temperatures between 125 and $135 \mathrm{~K}$ in a vacuum chamber $\left(5 \times 10^{-9} \mathrm{mbar}\right)$ by the method of Kouchi \& Kuroda (1990). In the present experiment the walls of the vacuum chamber were at room temperature rather than at 10 K as in Kouchi \& Kuroda (1990). During deposition, structural changes in the ice film were examined in situ by reflection electron diffraction $(20 \mathrm{keV})$.

Figures 1 and 2 (Plate L1) show the schematic structural change of the ice film and its corresponding reflection electron diffraction patterns, respectively. A halo diffraction pattern in Figure $2 a$ clearly shows that the deposited ice is amorphous. The halo pattern becomes progressively weaker with increasing thickness (Fig. 2b). On the other hand, the intensity of the diffraction pattern corresponding to cubic ice (ice $I_{c}$ ) becomes stronger (Fig. 2b) and is dominant in the thicker film (Fig. 2c). Even at the end of the deposition, the thinner part of the film remains amorphous (Fig. 2d). This observation shows that a critical transition thickness, $h_{c}$, from a- $\mathrm{H}_{2} \mathrm{O}$ to ice $I_{c}$ exists in all cases. Keeping $h<h_{c}$ we observed the occurrence of crys- tallization to occur at a temperature $T_{c}=140 \pm 2 \mathrm{~K}$ by warming up $\mathrm{a}-\mathrm{H}_{2} \mathrm{O}\left(1 \mathrm{~K}\right.$ minute $\left.{ }^{-1}\right)$ after it was deposited in the range $125-130 \mathrm{~K}$.

Although many investigators implicitly realized that the surface temperature of an $\mathrm{a}-\mathrm{H}_{2} \mathrm{O}$ film may exceed the transition temperature for various reasons (e.g., Pryde \& Jones 1952; Beaumont, Chihara, \& Morrison 1961), we report here the first direct and quantitative measurement of $h_{c}$. Our results show that the mixture of a- $\mathrm{H}_{2} \mathrm{O}$ and ice $I_{c}$ obtained by vapor deposition (Dowell \& Rinfret 1960; Narten, Venkatesh, \& Rice 1976; Mayer \& Pletzer 1987; Breukers 1991) is not homogeneously distributed, as had earlier been assumed, but consists of a layered structure, with the lower layer being $\mathrm{a}-\mathrm{H}_{2} \mathrm{O}$ and the upper one ice $I_{c}$.

\section{ANALYSIS AND RESULTS}

To estimate the conditions under which the surface temperature leads to crystallization of the deposited layer, we consider, simultaneously, thermal conduction to the substrate, net rate of radiation input from the external radiation field and energy of deposition. The temperature distribution in the ice film is governed by the quasi-steady state diffusion equation

$$
\frac{\partial}{\partial x}\left[\kappa(T) \frac{\partial T}{\partial x}\right]=0,
$$

where $\kappa(T)$ denotes the thermal conductivity of the ice film at a temperature $T$. The boundary conditions for solving equation (1) are as follows: (1) $T=T_{0}$ at the surface of the substrate $(x=0)$ where $T_{0}$ is the temperature of the substrate, and (2) heat balance at the surface $(x=h)$ given by the relation

$$
(1-\bar{A}) \sigma T_{R}^{4}-\bar{\epsilon} \sigma T_{h}^{4}-\left.\kappa \frac{\partial T}{\partial x}\right|_{x=h}+V L=0,
$$

where $\bar{A}$ is the Planck-averaged albedo of the sample at temperature $T_{R}, T_{R}$ the temperature of the ambient radiation field, $\bar{\epsilon}$ the Planck-averaged emissivity at temperature $T_{h}, \sigma$ the Stefan-Boltzmann constant, $V$ the deposition rate, and $L$ the heat of condensation per unit volume. The energy deposited by 


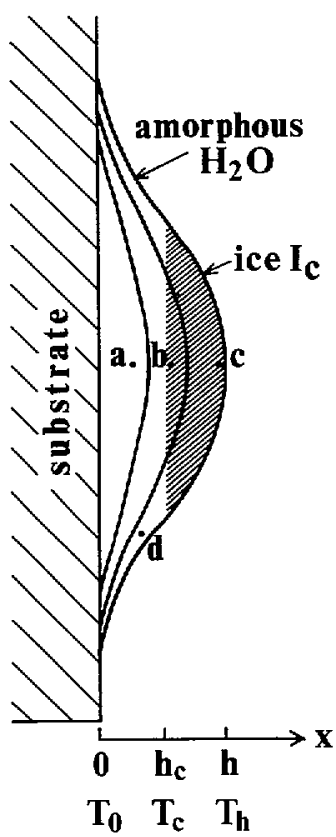

FIG. 1.-Schematic structural change of $\mathrm{H}_{2} \mathrm{O}$ thin film during deposition: substrate temperature, $T_{0}=134 \mathrm{~K}$; condensation rate, $V=1.4 \times 10^{-8} \mathrm{~cm}$ $\mathrm{s}^{-1}$; critical thickness, $h_{c}=0.3 \mu \mathrm{m}$. At thicknesses smaller than $h_{c}, \mathrm{a}-\mathrm{H}_{2} \mathrm{O}$ is deposited, while at thicknesses larger than $h_{c}$, crystalline ice, $I_{c}$, begins to be deposited (shaded part).

the impinging electrons $(20 \mathrm{keV})$ producing the diffraction patterns (Fig. 2) is sufficiently small to be neglected.

The solution of equation (1) with the boundary conditions (1) and (2) is given by

$$
h=\left(T_{h}-T_{0}\right) \bar{\kappa} /\left[(1-\bar{A}) \sigma T_{R}^{4}-\bar{\epsilon} \sigma T_{h}^{4}+V L\right],
$$

where

$$
\bar{\kappa} \equiv\left(T_{h}-T_{0}\right)^{-1} \int_{T_{0}}^{T_{h}} \kappa(T) d T
$$

is the mean thermal conductivity between $x=0$ and $x=h$ (or between $T_{0}$ and $T_{h}$ ).

To calculate an upper limit on $\bar{\kappa}$ we let $\bar{A} \sim 0, \bar{\epsilon} \sim 1$. The value of $L$ is taken to be represented by that for crystalline (hexagonal) ice, that is, $L=2.8 \times 10^{10} \mathrm{ergs}^{-3}$. As noted before, crystallization occurs at $T_{c}=140 \mathrm{~K}, h=h_{c}$. Table 1 shows the data used in the present study and the resulting values of $\bar{\kappa}$. Figure 3 shows the range of thermal conductivities of $\mathrm{a}-\mathrm{H}_{2} \mathrm{O}, \bar{\kappa}$, obtained in the present study. It is apparent that the data are scattered, but, in all cases, $\bar{\kappa}$ is much smaller than estimated by Klinger.

\section{DISCUSSION}

We suggest that the slow deposition procedure must be the cause of the material showing such a low thermal conductivity. This conjecture seems to be confirmed by the fact that, in general, using slower deposition rates tends to lead to much smaller derived values of $\bar{\kappa}$ - by a factor of 10,000 less than those estimated theoretically by Klinger (Fig. 4). Even for the highest deposition rates used by other experimenters, the value of $\bar{\kappa}$ is still at least 10 times smaller than that of Klinger.

The possibility that the extremely low thermal conductivity is a result of high porosity seems to be negated by the measure-

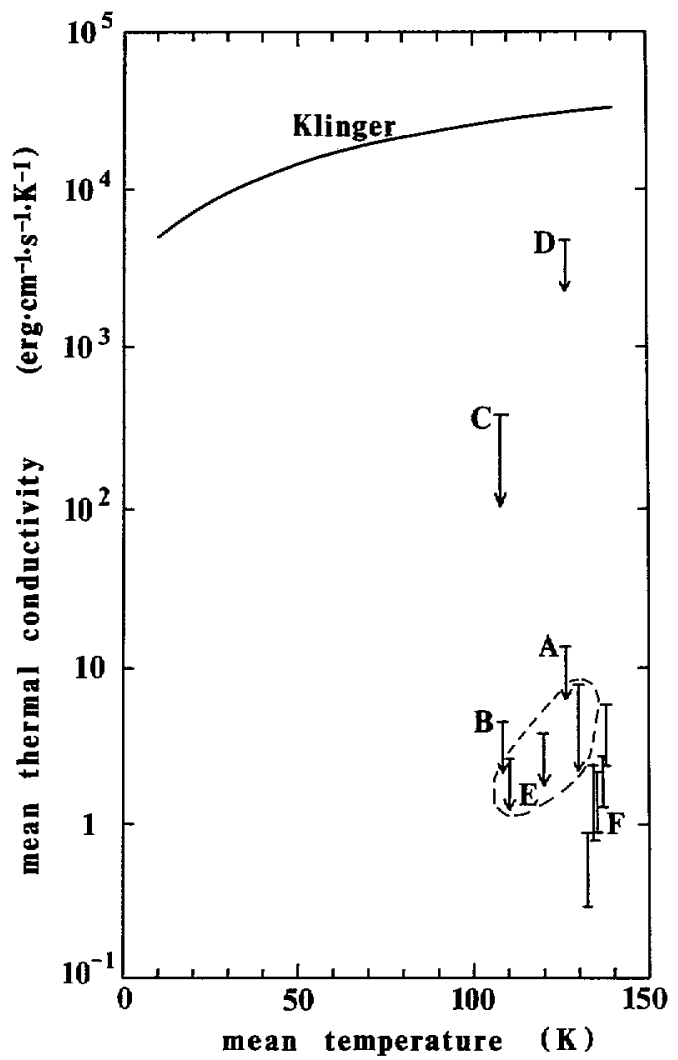

Fig. 3.-Mean thermal conductivities of a- $\mathrm{H}_{2} \mathrm{O}$ obtained in the present study (F), as well as those deduced from other experiments (A-E) and that estimated by Klinger (1980) (solid line). Points labeled A-F are the same as in Table 1. Note that the data from A to E show upper limits on $\bar{\kappa}$.

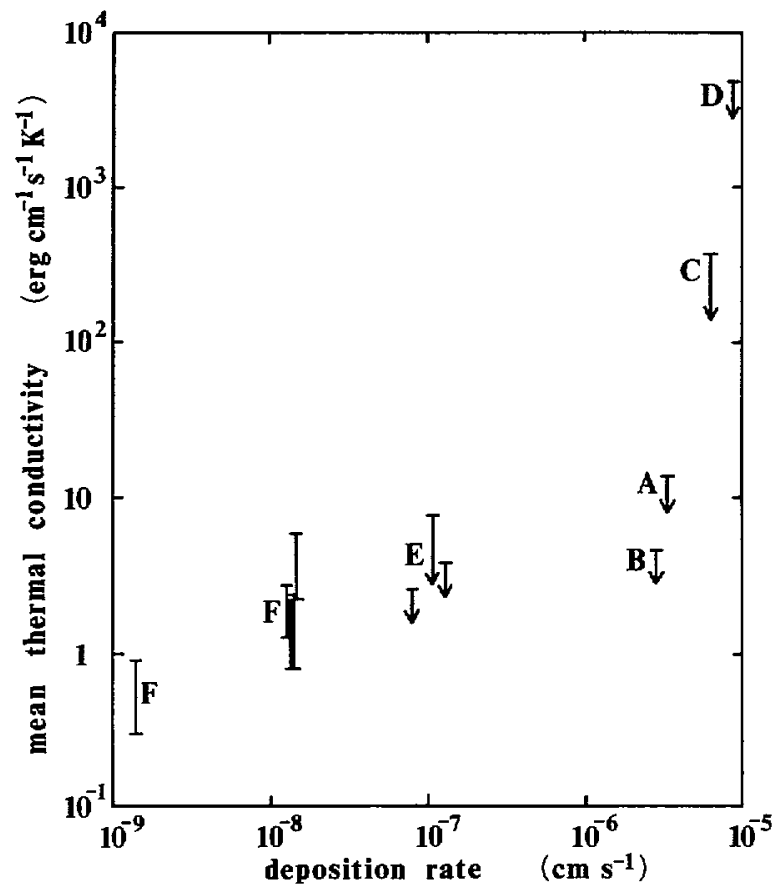

Fig. 4.-Mean thermal conductivity as a function of deposition rate. Points labeled A-F are the same as in Table 1. 
TABLE 1

Summary of the Experimental Data Used to Calculate $\bar{\kappa}$

\begin{tabular}{|c|c|c|c|c|c|c|c|}
\hline $\begin{array}{c}T_{0} \\
(\mathrm{~K})\end{array}$ & $\begin{array}{c}T_{R} \\
(\mathrm{~K})\end{array}$ & $\begin{array}{c}V \\
\left(\mathrm{~cm} \mathrm{~s}^{-1}\right)\end{array}$ & $\begin{array}{c}h_{c} \\
(\mu \mathrm{m})\end{array}$ & $\begin{array}{c}\bar{\kappa} \\
\text { (ergs cm } \\
\mathrm{s}^{-1} \mathrm{~K}^{-1} \text { ) }\end{array}$ & Method & Label $^{\mathrm{a}}$ & Notes $^{\mathrm{b}}$ \\
\hline $113 .$. & $\geq 113$ & $3.3 \times 10^{-6}$ & 60 & $<14$ & $\mathrm{XD}^{\mathrm{c}}$ & A & 1 \\
\hline $77 \ldots$ & $\geq 77$ & $\leq 2.8 \times 10^{-6}$ & $<50^{\mathrm{d}}$ & $<4.7$ & $\mathrm{XD}^{\mathrm{c}}$ & B & 2 \\
\hline $77 \ldots \ldots \ldots$ & $\geq 77^{\mathrm{d}}$ & $6.3 \times 10^{-6 d}$ & $<1500^{\mathrm{e}}$ & $<370$ & $\mathrm{XD}^{\mathrm{c}}$ & $\mathrm{C}$ & 3 \\
\hline $113 \ldots \ldots \ldots$ & $\sim 300$ & $8.8 \times 10^{-6}$ & $<1890$ & $<480$ & $\mathrm{XD}^{\mathrm{c}}$ & $\mathrm{D}$ & 4 \\
\hline $80 .$. & $\sim 300$ & $8 \times 10^{-8}$ & $<3.6$ & $<2.6$ & $\mathrm{IR}^{\mathrm{f}}$ & E & 5 \\
\hline $100 \ldots$ & $\sim 300$ & $1.3 \times 10^{-7}$ & $<3.5$ & $<3.9$ & $\mathrm{IR}^{\mathrm{f}}$ & E & 5 \\
\hline $120 \ldots \ldots \ldots$ & $\sim 300$ & $1.1 \times 10^{-7}$ & $<3.5$ & $<7.7$ & $\mathbf{I R}^{\mathrm{f}}$ & $\mathrm{E}$ & 6 \\
\hline $125 \ldots \ldots \ldots$ & $\sim 300$ & $1.4 \times 10^{-9}$ & $0.2 \pm 0.1$ & $0.6 \pm 0.3$ & $\mathrm{ED}^{8}$ & $\mathrm{~F}$ & 7 \\
\hline $129 \ldots \ldots \ldots$ & $\sim 300$ & $1.4 \times 10^{-8}$ & $0.4 \pm 0.2$ & $1.6 \pm 0.8$ & $\mathrm{ED}^{8}$ & $\mathrm{~F}$ & 7 \\
\hline $130 \ldots \ldots \ldots$ & $\sim 300$ & $1.4 \times 10^{-8}$ & $0.4 \pm 0.2$ & $1.5 \pm 0.7$ & $\mathrm{ED}^{8}$ & $\mathrm{~F}$ & 7 \\
\hline $134 \ldots \ldots \ldots$ & $\sim 300$ & $1.4 \times 10^{-8}$ & $0.3 \pm 0.1$ & $2.0 \pm 0.7$ & $\mathrm{ED}^{\mathrm{g}}$ & $\mathrm{F}$ & 7 \\
\hline $135 \ldots \ldots \ldots$ & $\sim 300$ & $1.4 \times 10^{-8}$ & $0.5 \pm 0.2$ & $4.1 \pm 1.8$ & $\mathrm{ED}^{8}$ & $\mathrm{~F}$ & 7 \\
\hline
\end{tabular}

NoTES-(1) Thickness of $60 \mu \mathrm{m}$ is the maximum obtainable for vitreous ice (Dowell \& Rinfret 1960). (2) Precrystalline ice (Olander \& Rice 1972). (3) Ice $I_{c}$ in the presence of a- $\mathrm{H}_{2} \mathrm{O}$ (Narten et al. 1976). (4) Largely a- $\mathrm{H}_{2} \mathrm{O}$ with a small amount of ice $I_{c}$ (Mayer \& Pletzer 1987). (5) Mixture of a- $\mathrm{H}_{2} \mathrm{O}$ and ice $I$ (Breukers 1991). (6) Mixture of a- $\mathrm{H}_{2} \mathrm{O}$ and ice $I$ but almost crystalline (Breukers 1991). (7) When sample thickness is smaller than $h_{c}$, deposited ice is a- $\mathrm{H}_{2} \mathrm{O}$. On the other hand, when sample is thicker than $h_{c}$, ice $I_{c}$ is deposited (present study).

a Points as labeled in Figs. 3 and 4.

${ }^{b}$ Description of deposit given by respective authors.

c X-ray diffiaction.

${ }^{d}$ Estimated value by present study.

' In the original paper (Narten et al. 1976), they gave $h=15 \mathrm{~mm}$, but it might be a misprint. Probably $h=1.5 \mathrm{~mm}$ is correct.

${ }^{f}$ Far-infrared spectra.

${ }^{\mathrm{g}}$ Reflection electron diffraction.

ment of the infrared absorptivity of similar samples which indicate a mean density $\sim 0.9 \mathrm{~g} \mathrm{~cm}^{-3}$ (e.g., Breukers 1991). An alternative justification for such small values of $\kappa$ is the existence of a connected network of microcracks, separting larger islands as observed in a-Ge (Donovan \& Heinemann 1971) and a-C (Jensen \& Hoffman 1991). It is possible, in such a configuration, to have an extremely small ratio of contact area to real surface area even with a low porosity. The islands may be connected by thin strands of a few water molecules.

\section{HEAT TRANSFER IN A COMET NUCLEUS}

Since the cometary nucleus is very porous (Greenberg \& Hage 1990; Rickman 1990), the thermal conductivity of the nucleus may be one to two orders of magnitude smaller than that of the constituent icy grains (Greenberg, Zhao, \& Hage 1989). This suggests that the net thermal conductivity of the comet nucleus is certainly smaller than 1 ergs $\mathrm{cm}^{-1} \mathrm{~s}^{-1} \mathrm{~K}^{-1}$ and possibly as low as $10^{-2} \mathrm{ergs} \mathrm{cm}^{-1} \mathrm{~s}^{-1} \mathrm{~K}^{-1}$ : a quite shocking result. With such a small value of $\kappa$, the mechanism of heat transfer in the comet nucleus on short time scales may not be only by conduction. Perhaps radiation transport or diffusion of evaporated icy grain mantle molecules may be important.

Since the interstellar grains absorb effectively in the visual and the phase function is highly forward throwing $(\langle g\rangle \sim 1)$, the optical depth in the visual at $1 \mathrm{~cm}$ is $\tau_{v}=n_{d} Q_{\mathrm{abs}} \pi a_{d}^{2}=10^{4}$, where $n_{d}$ is the number density of grains in the aggregate, $Q_{\text {abs }}$ is the absorption efficiency of an individual grain, and $a_{d}$ is a mean grain radius, so that no visual radiation penetrates directly beyond a depth of $1 \mu \mathrm{m}$. The optical depth at $10 \mu \mathrm{m}$ in the infrared is about $\tau_{10} \sim 0.05 \tau_{v}$ based on the interstellar extinction curve (Savage \& Mathis 1979) so that infrared energy transport is also quite small.
Next, we consider the diffusive heat transport by sublimated water molecules. Following the result of Mekler, Prialnik, \& Podolak (1990) for a porosity of 0.8 and approximately extrapolated to $\sim 1 \mu \mathrm{m}$ pore sizes which are relevant to the interstellar dust model (Greenberg \& Hage 1990), the inward flux of water in a comet of Halley size $(r=2.5 \mathrm{~km})$ at a depth of $1 \mathrm{~cm}$ is $\Phi \sim 10^{25}$ molecules $\mathrm{s}^{-1}$. An effective diffusive thermal conductivity is

$$
\kappa_{\mathrm{dif}}=\frac{\Phi \Delta E}{4 \pi r^{2}} \frac{d r}{d T}
$$

where $\Delta E$ is the total sublimation energy deposited per condensing molecule $\left(\sim 0.57 \mathrm{eV}\right.$ molecule $\left.{ }^{-1}\right)$ and $d T / d r$ is the temperture gradient. Immediately at the surface by thermal conductivity alone, the gradient $d T / d r$ is of the order of $(d H / d t) \kappa_{c}^{-1}$ where $d H / d t\left(2\right.$ calories $\mathrm{cm}^{-2}$ minutes $^{-1}$ at $\left.1 \mathrm{AU}\right)$ is the solar energy flux per unit area, and $\kappa_{c}$ the thermal conductivity of crystalline ice. From this we obtain for $r=2.5 \mathrm{~km}$ $\kappa_{\mathrm{dif}}=11.6(\mathrm{dH} / d t)^{-1} \kappa_{c}=8.4 \times 10^{-6} \kappa_{c} \simeq 0.84 \approx \bar{\kappa}$, which only changes the effective total $\kappa$ by a factor of 2 and therefore results in little change at $1 \mathrm{AU}$, even less at greater distances.

The more volatile molecules like $\mathrm{CO}$ and $\mathrm{CO}_{2}$ may be expected to play a more important role in diffusive heat transport than $\mathrm{H}_{2} \mathrm{O}$ (Smoluchowski 1985; Espinasse et al. 1991), but with the very small pore sizes their effect is probably reduced by trapping in recondensed $\mathrm{H}_{2} \mathrm{O}$. Furthermore, the amount of evaporation of $\mathrm{H}_{2} \mathrm{O}$ and release of trapped molecules (like $\mathrm{CO}$ and $\mathrm{CO}_{2}$ ) is clearly limited to a depth of less than several tens of centimeters for normal periodic comets (Greenberg et al. 1992). Consequently, it appears unlikely that the heating of a comet at depths larger than several tens of centimeters has ever occurred. 
Hans Rickman is thanked for his advice. We thank the Netherlands Organization for Scientific Research (NWO) and the Japan Society for the Promotion of Science (JSPS) for support under an exchange program. Part of this research was supported by NASA grant (NGR 33-018-148). One of the authors (T. Y.) acknowledges support from the University of Tsukuba, Japan. One of the authors (A. K.) was also supported by the Yamada Science Foundation, Japan.

Beaumont, R. H., Chihara, H., \& Morrison, J. A. 1961, J. Chem. Phys., 34, 1456 REFERENCES

Breukers, R. 1991, Ph.D. thesis, Univ. of Leiden

d'Hendecourt, L. B., Allamandola, L. J., \& Greenberg, J. M. 1985, A\&A, 152, 130

Donovan, T. M., \& Heinemann, K. 1971, Phys. Rev. Lett., 27, 1794

Dowell, L. G., \& Rinfret, A. P. 1960, Nature, 188, 1144

Espinasse, S., Klinger, J., Ritz, C., \& Schmitt, B. 1991, Icarus, 92, 350

Greenberg, J. M. 1982, in Comets, ed. L. L. Wilkening (Tucson: Univ. Arizona Press), 131

Greenberg, J. M., \& Hage, J. 1990, ApJ, 361, 260

Greenberg, J. M., Yamamoto, T., \& Xing, Z. F. 1992, ApJL, submitted

Greenberg, J. M., Zhao, N., \& Hage, J. 1989, Ann. Phys. Fr., 14, 103

Jensen, C. L., \& Hoffman, R. A. 1991, J. Vac. Sci. Technol. A., 8, 116

Klinger, J. 1980, Science, 209, 271

Kouchi, A., \& Kuroda, T. 1990, Nature, 344, 134

Mayer, E., \& Pletzer, R. 1987, J. Phys. (Paris), 48, C1, 581

Mekler, Y., Prialnik, D., \& Podolak, M. 1990, ApJ, 356, 682

Narten, A. H., Venkatesh, C. G., \& Rice, S. A. 1976, J. Chem. Phys., 64, 1106

Olander, D. S., \& Rice, S. A. 1972, Proc. Nat. Acad. Sci., 69, 98

Pryde, J. A., \& Jones, G. O. 1952, Nature, 170, 685

Rickman, H. 1990, in Comet Halley, Investigations, Results, Interpretations. Vol. 2: Dust, Nucleus, Evolution, ed. J. W. Mason (New York: Ellis Horwood), 163

Savage, B. D., \& Mathis, J. S. 1979, ARA\&A, 17, 73

Smoluchowski, R. 1985, in Ices in the Solar System, ed. J. Klinger, D. Benest, A. Dollfus, \& R. Smoluchowski (Dordrecht: Reidel), 397 

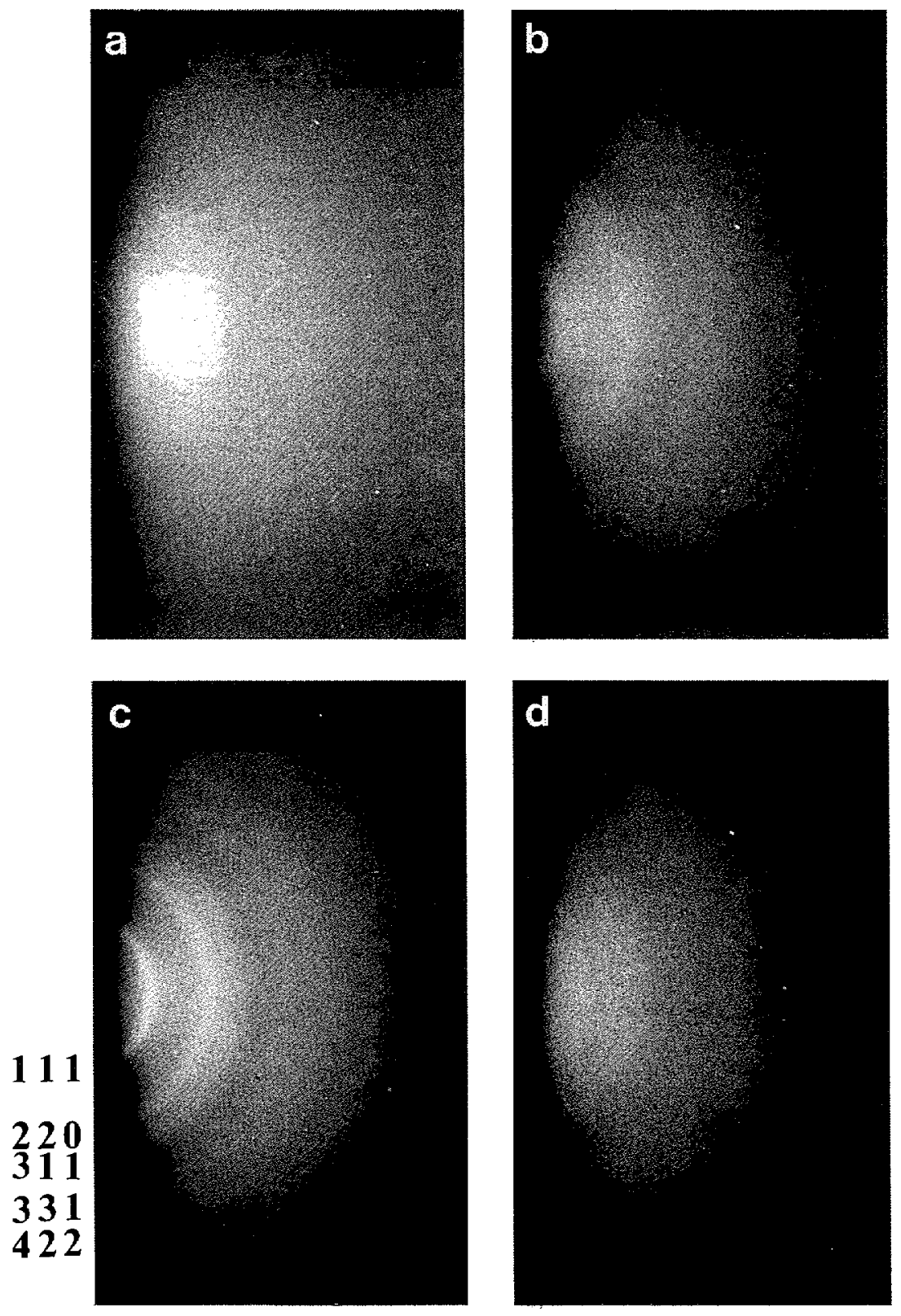

FIG. 2.-Reflection electron diffraction patterns of ice film: $(a)-(d)$ correspond to structural changes indicated in Fig. 1. Kouchi et al. (see 388, L73) 